\title{
Influence of the gas-surface interaction model on time-dependent rarefied gas simulations
}

\author{
Nemanja Andric*, M. Hossein Gorji, Patrick Jenny \\ Institute of Fluid Dynamics, ETH Zürich, Sonneggstrasse 3, 8092 Zürich, Switzerland
}

\section{Abstract}

In this paper, the influence of the gas-surface interaction on the time-dependent rarefied gas flow through a short tube into vacuum is investigated. Due to a significant scale separation, the flow is simulated using a hybrid scheme proposed by Vargas et al. $[1,2]$, in which the pressure change in the upstream chamber is coupled to the flow rate obtained by the Direct Simulation Monte Carlo (DSMC) computations. First, the influence of gas-surface interaction is demonstrated through comparison of DSMC simulation results obtained for different values of accommodation coefficients. The obtained dataset is then used to show how the boundary conditions can affect the time-dependent gas flow, even when applied on the small surface area of the device. It is argued that the domain of possible solutions is bounded by the cases of specular and diffuse scattering. Furthermore, it is investigated how this domain can be affected by measurement uncertainties present in the experimental setup. Next, the analysis of the gas-surface interaction is extended to binary mixtures. In the end, it is discussed how the obtained results can be used in order to improve the efficiency of gas separation mechanisms.

Keywords: Rarefied gas dynamics, Time-dependent flow, DSMC, Hybrid modelling, Gas-surface interaction, Gas separation mechanism

\footnotetext{
*Corresponding author:

Email address: andricn@ifd.mavt.ethz.ch (Nemanja Andric)

Preprint submitted to Vacuum

March 31, 2016
} 


\section{Introduction}

Rarefied gas flows are a prominent feature in many industrial areas ranging from vacuum technologies to aerospace engineering. Particularly in the former, rapid pressure change of the gas is of significant practical interest. These include leak testing, CD/DVD metallization, surface coating, mass spectrometry and vacuum gauge testing, among others. In these applications, the flow field is mostly timedependent, which significantly increases the complexity of the required computer simulations and experimental setups.

Recently, a hybrid approach for simulating time-dependent rarefied gas flows was introduced by Vargas et al. [2]. In their setup, the gas expands from a high pressure chamber towards a low pressure one through a conducting tube. Since in such a scenario the time scale of the gas flow through the tube is significantly lower than the time scale of the pressure change, the kinetic simulation of the entire domain becomes computationally prohibitive. In the introduced hybrid scheme, kinetic modelling (micro model) is employed only for a small region around the tube, while the gas behaviour in the rest of the domain is modelled using mass conservation principle together with the equation of state (macro model). The information from the micro model is fed into the macro model in the form of the mass flow rate through the tube, whereas the pressure change in the chambers is obtained from the equation of state.

In a follow-up study [1], the authors investigated the time-dependent rarefied gas flow of single gases and binary mixtures from an upstream vessel through a short tube into vacuum using the above-mentioned hybrid scheme. Through the comparison of their simulation results and the experimental data from the modified setup of $[8,9,10]$, the authors reported a discrepency of $10 \%$ for the gas pressure. 
The main cause of the discrepancy was attributed to the geometrical uncertainties of the flow configuration. For modelling gas-surface interaction, the diffuse scattering kernel was used.

Due to its simplicity and acceptable accuracy, the diffuse scattering kernel is broadly used for describing gas-surface interactions. Although it is an acceptable choice for many practical computations, it has been observed that in certain test cases the numerical data obtained by the diffuse kernel cannot match the corresponding experimental results (see e.g. [14],[15], [16]). More accurate simulations can be performed by using the diffuse-specular Maxwell kernel [33] or the Cercignani-Lampis (CL) kernel [11]. Unlike the Maxwell kernel, the CL kernel is able to reproduce physical lobular patterns for monatomic gases and it gives more accurate predictions of the flow field [14]. Furthermore, in different studies concerning the influence of the gas-surface interaction on the rarefied gas flow through a short channel [17] and tube [21] into vacuum, it has been shown that the mass flow rate strongly depends on the value of the tangential momentum accommodation coefficient (TMAC).

Here, a systematic study of the influence of gas-surface interaction on the timedependent rarefied gas flow is carried out using the above-mentioned hybrid approach. A brief overview of the flow configuration and the hybrid scheme is given in $§ 2$. Next, the scattering kernels employed for modelling of the gas-surface interaction are described in $\S 3$. The results are presented and analysed in $\S 4$, where the influence of accommodation coefficients and geometrical uncertainties on the flow is investigated. In the end, some concluding remarks are given in $\S 5$.

\section{Hybrid modelling}

In this section, a brief overview of the hybrid scheme will be given, while all additional information about the scheme and flow configuration can be found in $[1,2]$. 


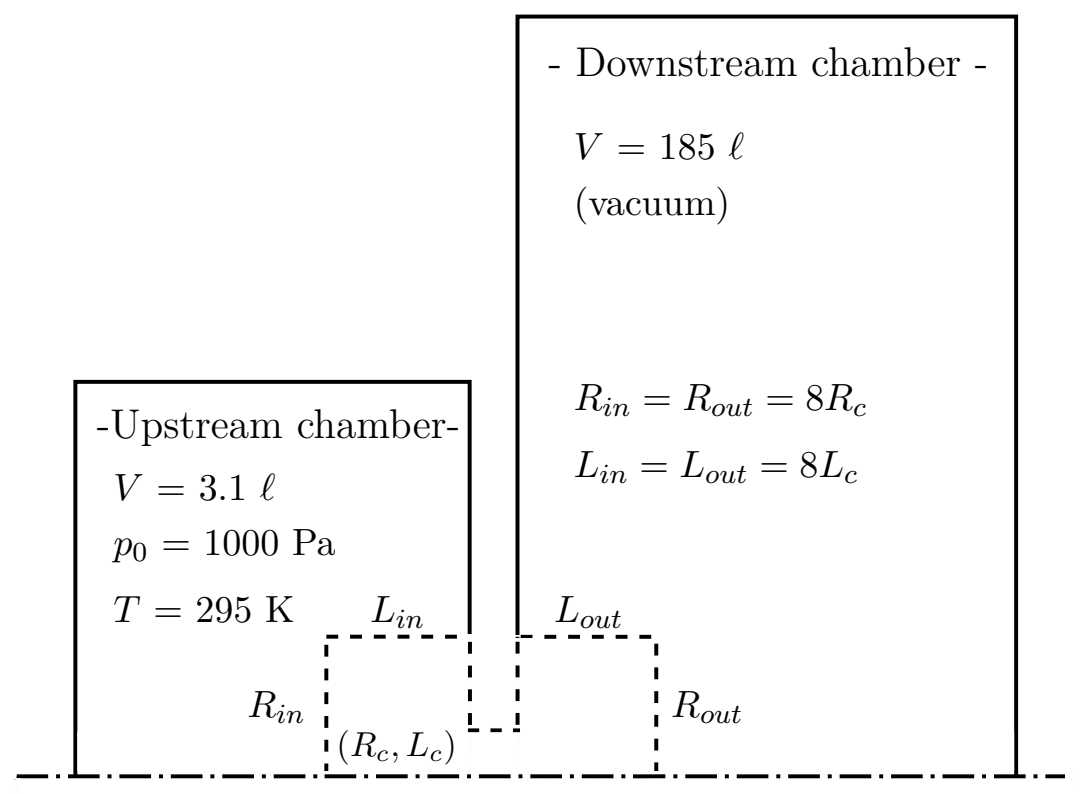

Figure 1: Schematic of the gas expansion setup of [1]. Dashed line outlines the area where DSMC is employed.

The system under consideration consists of two cylindrical chambers connected via a short tube (schematic view given in Fig. 1) and it is identical to the one investigated in [1]. The gas in the upstream chamber is initially at pressure $p_{0}=1000 \mathrm{~Pa}$ and temperature $T=295 \mathrm{~K}$, while the downstream chamber is held at vacuum. The reported volumes of the upstream and downstream chambers are $3.1 \ell$ and $185 \ell$, respectively, and the connecting tube has the length $L=0.4962 \pm 0.01 \mathrm{~mm}$ and diameter $D=2 R=1.0113 \pm 0.005 \mathrm{~mm}$. Hence, the tube volume is negligible compared to the volume of the chambers. This causes a significant scale separation of the characteristic flow times through the tube and inside the reservoir. In other words, the time step necessary to resolve the flow through the tube would be many orders of magnitude smaller compared to the gas relaxation time in the upstream 
chamber. For this reason, a full kinetic description of the gas expansion would be computationally impractical. The computational efficiency can be improved by implementing the hybrid scheme [2], in which the kinetic description is employed solely for the quasi-steady gas flow through the tube (micro model). Then, the relaxation of the gas in the upstream chamber is described by using the equation of state together with the mass conservation principle (macro model).

The derivation of the macro model is discussed first. The following formulation is laid down for the general case of the binary gas mixture and simplified expressions for the single gas can be readily deduced. Since only expansion of monatomic gases is investigated, the gas state in the upstream chamber can be described by the ideal gas law

$$
p_{i}(t) V=N_{i}(t) \Re T \quad(i \in\{1,2\})
$$

where $p_{i}(t)$ and $N_{i}(t)$ are the partial pressure and number of moles of species $i$ at time $t$, respectively, $\Re$ is the universal gas constant with the value of $8.314 \mathrm{~J} / \mathrm{mol} / \mathrm{K}$ and $V$ is the chamber volume. The total pressure $p(t)$ and total number of moles $N(t)$ in the mixture are obtained by summing the respective values of these quantities for each species. The pressure is assumed to be uniform in the entire upstream chamber. Furthermore, since the gas expansion is not very rapid, the temperature of the gas $T$ is assumed to remain constant throughout the process, which is verified by measurements (see [1]).

The amount of the lighter species in the mixture is specified by the molar fraction

$$
C(t)=\frac{N_{1}(t)}{N_{1}(t)+N_{2}(t)}=\frac{p_{1}(t)}{p_{1}(t)+p_{2}(t)}
$$


and the degree of gas rarefaction is determined by the rarefaction parameter

$$
\sigma(t)=\frac{p(t) R}{\mu(t) v(t)}
$$

where $\mu(t)$ is the mixture viscosity, $v(t)=\sqrt{2 \Re T / M(t)}$ is the most probable molecular velocity and $M(t)$ is the molar mass of the mixture. Note that the value of the rarefaction parameter is inversely proportional to the Knudsen number.

The amount of gas in the upstream chamber decreases in time due to the outflow through the tube. The temporal evolution of the number of moles of species $i$ in the upstream chamber is expressed as

$$
\frac{d N_{i}}{d t}=-\dot{N}_{i}(t)
$$

where $\dot{N}_{i}(t)$ is the molar flow rate of species $i$ through the tube. Differentiation in time of the ideal gas law (1), together with the mass conservation equation (4), results in the pressure evolution equation of the form

$$
\frac{d p_{i}}{d t}=-\dot{N}_{i}(t) \frac{\Re T}{V}
$$

which directly links the change of pressure in the upstream chamber to the molar flow rate through the tube. As an additional remark, the downstream chamber is maintained at vacuum during the expansion process.

The molar flow rate through the tube is estimated by using an appropriate kinetic description of the pressure driven flow. Rarefied gas flow through a cylindrical tube has been extensively studied in the past as one of the benchmark cases for validation of different kinetic schemes $[19,21,22,23,24,25,26]$. In the present study, simula- 
tions have been performed by using the 'No Time-Counter' version of Bird's DSMC method [3] for hard sphere molecules. The computational domain consists of the tube of radius $R_{c}=0.50565 \mathrm{~mm}$ and length $L_{c}=0.4964 \mathrm{~mm}\left(L_{c} / R_{c} \approx 1\right)$, together with the inlet $\left(R_{\text {in }}, L_{\text {in }}\right)$ and outlet $\left(R_{\text {out }}, L_{\text {out }}\right)$ cylindrical volumes connected to the tube ends. The size of these volumes is much smaller than the respective size of the upstream and downstream chambers, but still large enough to ensure uniform conditions at the domain boundaries $\left(R_{\text {in }}=R_{\text {out }}=8 R_{c}, L_{\text {in }}=L_{\text {out }}=8 L_{c}\right)$.

Here, instead of performing a new DSMC simulation at each macroscopic time step, a suitable DSMC database for different values of rarefaction parameter $(\sigma=0$, $0.1,0.5,1,5,10,25,50,75,100)$ and molar fraction in the case of mixture $(C=0$, $0.125,0.25,0.375,0.5,0.625,0.75,0.875,1)$ has been precomputed in the same way as in [1]. The solution procedure can then be summarized as follows:

1. At each instance in time $t_{j}$, partial pressure $p_{i}\left(t_{j}\right)$ is known from the previous time step. The number of moles $N_{i}\left(t_{j}\right)$ of each species, the molar fraction $C\left(t_{j}\right)$ and the rarefaction parameter $\sigma\left(t_{j}\right)$ are determined from Eqs. (1), (2) and (3), respectively.

2. The molar flow rate $\dot{N}_{i}\left(t_{j}\right)$ of each species is computed by interpolating between the corresponding values from the precomputed DSMC database for $C\left(t_{j}\right)$ and $\sigma\left(t_{j}\right)$.

3. Equation (5) is numerically integrated in time for each species, which gives $p_{i}\left(t_{j}+\Delta t\right)$ at time $t_{j}+\Delta t$, where $\Delta t$ is an appropriate macroscopic time step.

4. The procedure is repeated for new partial pressure $p_{i}\left(t_{j}+\Delta t\right)$ by going back to Step 1. The simulation is continued until the desired macroscopic time is reached.

The described procedure is implemented for simulating expansion of the single 
gases $\mathrm{He}, \mathrm{Ne}, \mathrm{Ar}$ and $\mathrm{Kr}$ and binary mixture of $\mathrm{He}$ and Ar into vacuum under the specified flow conditions $\left(p_{0}=1000 \mathrm{~Pa}, T=295 \mathrm{~K}\right)$. Different types of boundary conditions employed in micromodel are summarised in $\S 3$.

\section{Gas-surface interaction}

Two essential scenarios characterize the gas-surface interaction. One is specular scattering, where the particle reflection from the surface is mirror-like and without any energy exchange. If the particle pre- and post-collision velocities are realizations from the sample spaces $\mathbf{v}^{\prime}=\left(v_{t 1}{ }^{\prime}, v_{t 2}{ }^{\prime}, v_{n}{ }^{\prime}\right)$ and $\mathbf{v}=\left(v_{t 1}, v_{t 2}, v_{n}\right)$, respectively, then the specular scattering kernel (distribution function) reads

$$
f^{s p e}\left(\mathbf{v} \mid \mathbf{v}^{\prime}\right)=\delta\left(\mathbf{v}^{\prime}-\left(\mathbf{v}-2 v_{n} \mathbf{n}\right)\right) ; \quad v_{n} \geq 0,
$$

where $\delta()$ is the Dirac delta function, $v_{n}$ is the normal velocity component and $\mathbf{n}$ is the unit vector normal to the surface. By definition, the unit vector $\mathbf{n}$ points into the computational domain and therefore $v_{n} \geq 0$ and $v_{n}^{\prime} \leq 0$. Although the specular kernel gives a simple and straightforward way to model gas-surface interaction, in reality full specular scattering will never occur. For this to happen, the surface should be infinitely dense at the molecular level and perfectly smooth, which is impossible to occur in practical applications.

The opposite scenario is diffuse scattering, which is characterized by complete accommodation of particle energy. From the physical aspect, the particle is trapped by the surface molecules during the collision and after a certain time it is released with a random velocity depending on the wall temperature. In modelling of diffuse scattering, the surface can be treated as an interface between the computational and a virtual domain at equilibrium. The diffuse scattering kernel is defined by 
the marginal velocity distribution of the particles crossing the interface into the computational domain, i.e.

$$
f^{d i f f}\left(\mathbf{v} \mid \mathbf{v}^{\prime}\right)=\frac{v_{n}}{2 \pi \beta^{4}} \exp \left(-\frac{\mathbf{v} \cdot \mathbf{v}}{2 \beta^{2}}\right) ; \quad v_{n} \geq 0
$$

where $\beta=\sqrt{k T_{s} / m}$ is the thermal speed, $k$ is the Boltzmann constant, $T_{s}$ the surface temperature and $m$ the molecular mass. The diffuse scattering kernel has been successfully employed for modelling of gas-surface interactions in many problems. However, its applicability is not universal. For some problems, such as rarefied gas flow through channels and tubes, discrepancies between the computational and experimental results are observed if the diffuse kernel is applied [14].

The disagreement between the experiments and computation can be reduced, if a more general scattering kernel is employed. Optimally, such a kernel should be able to combine the elements of both specular and diffuse scattering. The straightforward way of constructing it is by direct coupling of the specular and diffuse kernels presented above. This gives the Maxwell scattering kernel based on Eqs. (6) and (7), which reads

$$
f^{M}\left(\mathbf{v} \mid \mathbf{v}^{\prime}\right)=\left(1-\alpha_{M}\right) \delta\left(\mathbf{v}^{\prime}-\left(\mathbf{v}-2 v_{n} \mathbf{n}\right)\right)+\alpha_{M} \frac{v_{n}}{2 \pi \beta^{4}} \exp \left(-\frac{\mathbf{v} \cdot \mathbf{v}}{2 \beta^{2}}\right) ; \quad v_{n} \geq 0
$$

where the coefficient $\alpha_{M}$ specifies the amount of particles which undergo diffuse reflection. Although its application typically gives a better match with the experimental data compared to both specular and diffuse kernels, the Maxwell kernel suffers from some inaccuracies. Most importantly, it is not able to reproduce physical lobular patterns of scattered molecules. A further issue exists with the generalization to polyatomic molecules, where the energy exchange between different modes of motion 
occurs during the interaction with the surface.

A more suitable model which allows transition between specular and diffuse scattering was introduced in 1971 by Cercignani and Lampis [11]. The Cercignani-Lampis (CL) kernel is based on mathematical reasoning with the aim of satisfying the reciprocity condition and producing the physical lobular patterns. It is given by the following expression

$$
\begin{aligned}
& f^{C L}\left(\mathbf{v} \mid \mathbf{v}^{\prime}\right)=\frac{v_{n}}{2 \pi \beta^{4} \alpha_{n} \alpha_{t}\left(2-\alpha_{t}\right)} \exp \left(-\frac{\left(\mathbf{v}_{\mathbf{t}}-\sqrt{1-\alpha_{t}} \mathbf{v}_{\mathbf{t}}^{\prime}\right)^{2}}{2 \beta^{2} \alpha_{t}\left(2-\alpha_{t}\right)}\right) \\
& \quad \times I_{0}\left(\frac{\sqrt{1-\alpha_{n}} v_{n}^{\prime} v_{n}}{\beta^{2} \alpha_{n}}\right) \exp \left(-\frac{v_{n}^{2}+\left(1-\alpha_{n}\right) v_{n}^{\prime 2}}{2 \beta^{2} \alpha_{n}}\right) ; \quad v_{n} \geq 0,
\end{aligned}
$$

where $\mathbf{v}_{\mathbf{t}}$ is the tangential velocity vector and $I_{0}$ is the zeroth order modified Bessel function of the first kind. The parameters $\alpha_{t}$ and $\alpha_{n}$ represent the accommodation coefficients of the tangential momentum and normal kinetic energy, respectively. In the case of specular scattering, the accommodation coefficients are zero, i.e. $\alpha_{t}=$ $\alpha_{n}=0$. On the other end, diffuse scattering is recovered for $\alpha_{t}=\alpha_{n}=1$. Moreover, the CL kernel also allows for backscattering with $\alpha_{t}=2$ and $\alpha_{n}=0$. Due to its ability to reproduce physical lobular patterns, the CL kernel gives more reliable and physically more accurate predictions of the flow field compared to the Maxwell scattering kernel $[14,15,16]$. Furthermore, it has been extended for the case of diatomic molecules with rotational degrees of freedom [12], [13]. 


\section{Results and discussion}

\subsection{DSMC results}

For DSMC calculations, a uniform mesh with 632'100 cells is employed (560x560 cells for both inner and outer cylinders and 70x70 cells for the tube). Since the problem is axisymmetric, a uniform particle distribution is achieved by splitting the computational domain into ten radial weighting zones and using a suitable algorithm for the particle number control. The total number of particles for the case of single gases is around $10^{7}$, whereas for the mixture is around $1.6 \times 10^{7}$. The time step size is restricted to half of the mean collision time, i.e. $\Delta t \leq 0.5 \mu / p_{0}$ and the simulations are run until the termination criterion $\sqrt{N_{\text {in }}} /\left(N_{\text {in }}+N_{\text {out }}\right)<0.002$ is reached $\left(N_{\text {in }}\right.$

Table 1:

DSMC database of dimensionless flow rates $J$ for single gases as a function of rarefaction parameter $\sigma \in[0,100]$ and $\alpha_{t}$. Gas-surface interaction is modelled with the CL kernel using $\alpha_{n}=1$.

\begin{tabular}{l|lllll}
\hline \hline$\sigma$ & $\alpha_{t}=0$ & $\alpha_{t}=0.25$ & $\alpha_{t}=0.5$ & $\alpha_{t}=0.75$ & $\alpha_{t}=1$ \\
\hline 0 & 0.283 & 0.245 & 0.225 & 0.207 & 0.191 \\
0.1 & 0.283 & 0.247 & 0.227 & 0.210 & 0.193 \\
0.5 & 0.292 & 0.261 & 0.239 & 0.221 & 0.203 \\
1 & 0.302 & 0.276 & 0.253 & 0.233 & 0.215 \\
5 & 0.355 & 0.333 & 0.310 & 0.289 & 0.271 \\
10 & 0.378 & 0.356 & 0.334 & 0.317 & 0.302 \\
25 & 0.396 & 0.381 & 0.364 & 0.350 & 0.342 \\
50 & 0.407 & 0.393 & 0.381 & 0.371 & 0.364 \\
75 & 0.410 & 0.399 & 0.389 & 0.382 & 0.378 \\
100 & 0.412 & 0.401 & 0.395 & 0.389 & 0.383 \\
\hline \hline
\end{tabular}


and $N_{\text {out }}$ are the total numbers of particles entering and exiting the tube inlet, respectively).

The same boundary condition is applied on all surfaces present in the DSMC simulation. The molar flow rates of single gases for the CL and Maxwell kernels are summarised in Tables 1 and 2, showing the dimensionless quantity $J$ defined as

$$
J_{i}(t)=\frac{\Re T}{\pi R^{2} v(t) p(t)} \dot{N}_{i}(t) \quad(i \in\{1,2\}) .
$$

Changing the value of $\alpha_{t}$ proved to have a significant impact on the resulting flow rates. On the other hand, the simulation results are independent of the value of $\alpha_{n}$, due to horizontal streamlines inside the tube and the assumed isothermal nature of

Table 2:

DSMC database of dimensionless flow rates $J$ for single gases as a function of rarefaction parameter $\sigma \in[0,100]$ and accommodation coefficient. Gas-surface interaction is modelled with the CL kernel using $\alpha_{n}=0$ and Maxwell kernel.

\begin{tabular}{l|cccccc}
\hline \hline$\sigma$ & $\alpha_{t}=0$ & $\alpha_{t}=0.5$ & $\alpha_{t}=1$ & $\alpha_{M}=0.25$ & $\alpha_{M}=0.5$ & $\alpha_{M}=0.75$ \\
\hline 0 & 0.282 & 0.224 & 0.192 & 0.254 & 0.231 & 0.210 \\
0.1 & 0.282 & 0.227 & 0.194 & 0.257 & 0.234 & 0.212 \\
0.5 & 0.292 & 0.239 & 0.202 & 0.266 & 0.244 & 0.223 \\
1 & 0.301 & 0.253 & 0.213 & 0.278 & 0.254 & 0.234 \\
5 & 0.354 & 0.310 & 0.271 & 0.331 & 0.308 & 0.288 \\
10 & 0.378 & 0.338 & 0.304 & 0.356 & 0.336 & 0.318 \\
25 & 0.399 & 0.367 & 0.343 & 0.381 & 0.364 & 0.352 \\
50 & 0.411 & 0.383 & 0.367 & 0.395 & 0.382 & 0.372 \\
75 & 0.413 & 0.391 & 0.379 & 0.401 & 0.390 & 0.383 \\
100 & 0.417 & 0.396 & 0.384 & 0.404 & 0.394 & 0.388 \\
\hline \hline
\end{tabular}


the flow. The discrepancy between the CL results for $\alpha_{n}=0$ and $\alpha_{n}=1$ is depicted in Fig. 2a. It can be seen that the average relative difference does not exceed $1 \%$ for
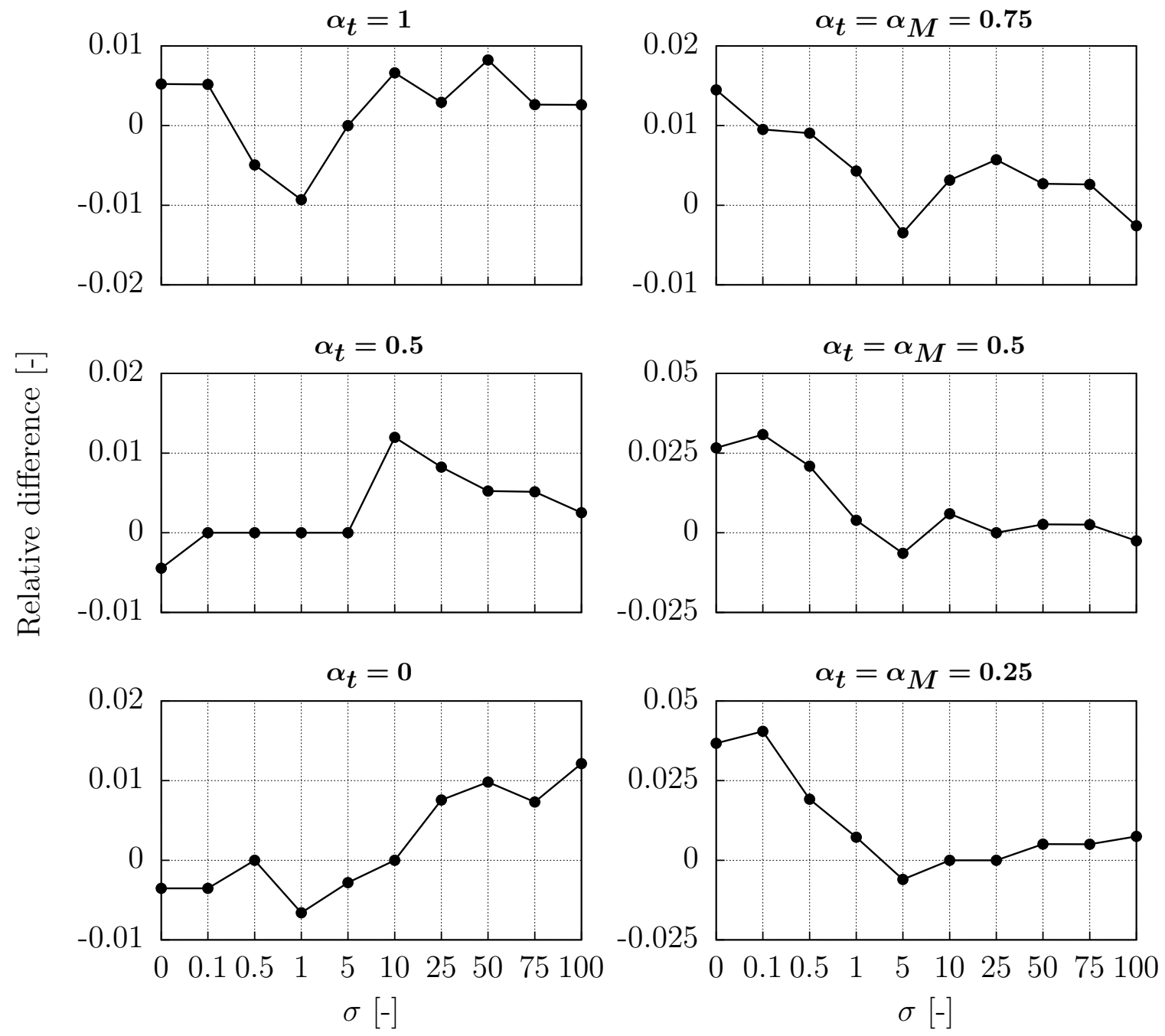

(a)

(b)

Figure 2: Comparison of DSMC results obtained with different scattering kernels. (a) Relative difference in $J$ between the CL data using $\alpha_{n}=0$ and $\alpha_{n}=1$ (with respect to the latter) (b) Relative difference in J between the Maxwell and CL data using $\alpha_{n}=1$ (with respect to the latter) 
the considered set of data, which is in the order of the expected numerical uncertainty.

Furthermore, the Maxwell data is compared with the CL results for $\alpha_{n}=1$ and the relative difference is depicted in Fig. 2b. While the discrepancy is negligible for $\sigma \geq 1$, it becomes more pronounced as the flow moves towards the free molecular regime $(\sigma=0)$. For $\alpha_{t}=\alpha_{M}=0.25$ and $\sigma \approx 0-0.1$, the flow rate based on the Maxwell kernel exceeds the one based on the CL kernel by approximately 4\%. For $\alpha_{t}=\alpha_{M}=0.5$ the difference is approximately $3 \%$ and it is further reduced to $1.5 \%$ for $\alpha_{t}=\alpha_{M}=0.75$. Therefore, one may infer that the Maxwell scattering kernel overestimates the flow rate in the collisionless regime compared to the CL kernel, which is in accordance with the results reported in [17]. Since the CL kernel presents a more realistic scattering model due to its ability to reproduce the physical lobular patterns, the flow rates based on the CL kernel are chosen as a reference dataset to carry out the macroscopic analysis of the flow.

Molar flow rates for the mixture of $\mathrm{He}$ and Ar in the limits of specular and diffuse scattering are provided in the Appendix. Note that the dimensionless flow rates are independent of the type of gas used in the simulations, since the gas-gas interactions are modelled with the hard sphere approximation, and therefore the obtained datasets are valid for any species. It is important to mention as well that our DSMC results for diffuse scattering agree within the error margin of $1 \%$ with the corresponding results reported in [1].

To highlight the influence of the gas-surface interaction, dimensionless flow rates for the case of specular $\left(\alpha_{t}=0\right)$ and diffuse scattering $\left(\alpha_{t}=1\right)$ are plotted in Fig. 3. For $\sigma=100$, the difference is around $7 \%$ and it increases at higher Knudsen numbers, reaching the upper bound of $32 \%$ for $\sigma=0$. Additionally in Fig. 3, the same flow rates are depicted as a function of $\alpha_{t}$ for different values of $\sigma$. From this plot, one can infer almost a linear relationship between $J$ and $\alpha_{t}$ for $\alpha_{t}>0.25$. This 
can be useful in estimating $J$ for an arbitrary $\sigma$, as long as the values of $J$ are known for $\alpha_{t}=0.25$ and 1 .

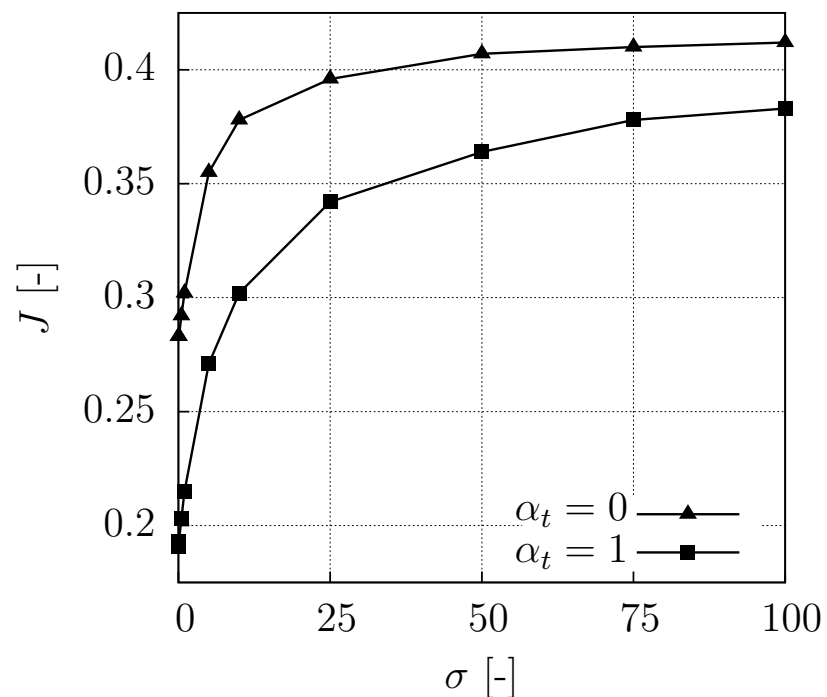

(a)

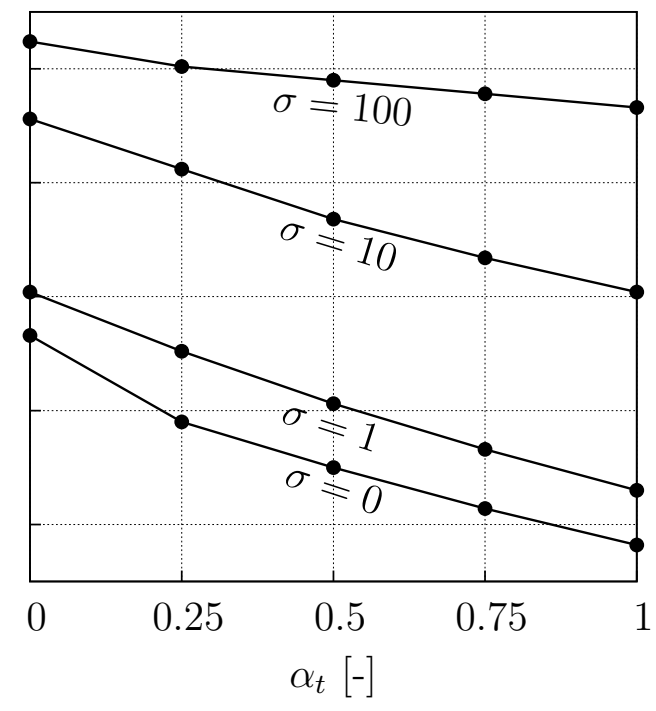

(b)

Figure 3: Dimensionless flow rate $J$ obtained with the CL kernel using $\alpha_{n}=1$ as a function of (a) rarefaction parameter $\sigma$ and (b) accommodation coefficient $\alpha_{t}$

\subsection{Expansion of single gases}

Pressure drop in the upstream chamber for $\mathrm{He}, \mathrm{Ne}, \mathrm{Ar}$ and $\mathrm{Kr}$ is calculated using the data given in Table 1. The results obtained for the case of complete specular and diffuse scattering are given in Fig. 4. Analysing the respective pressure profiles, a significant discrepancy between the two curves can be observed. For the case of specular scattering, the pressure drop is more rapid which results in shorter discharge time compared to diffuse scattering. More specifically, the pressure drop to $10 \mathrm{~Pa}$ is delayed by approximately $12 s, 23 s, 25 s$ and $34 s$ for $\mathrm{He}, \mathrm{Ne}, \mathrm{Ar}$ and $\mathrm{Kr}$, respectively, if diffuse scattering is used. This serves to highlight the significant impact boundary conditions can have on the recovered results of time-dependent flows, even when they 

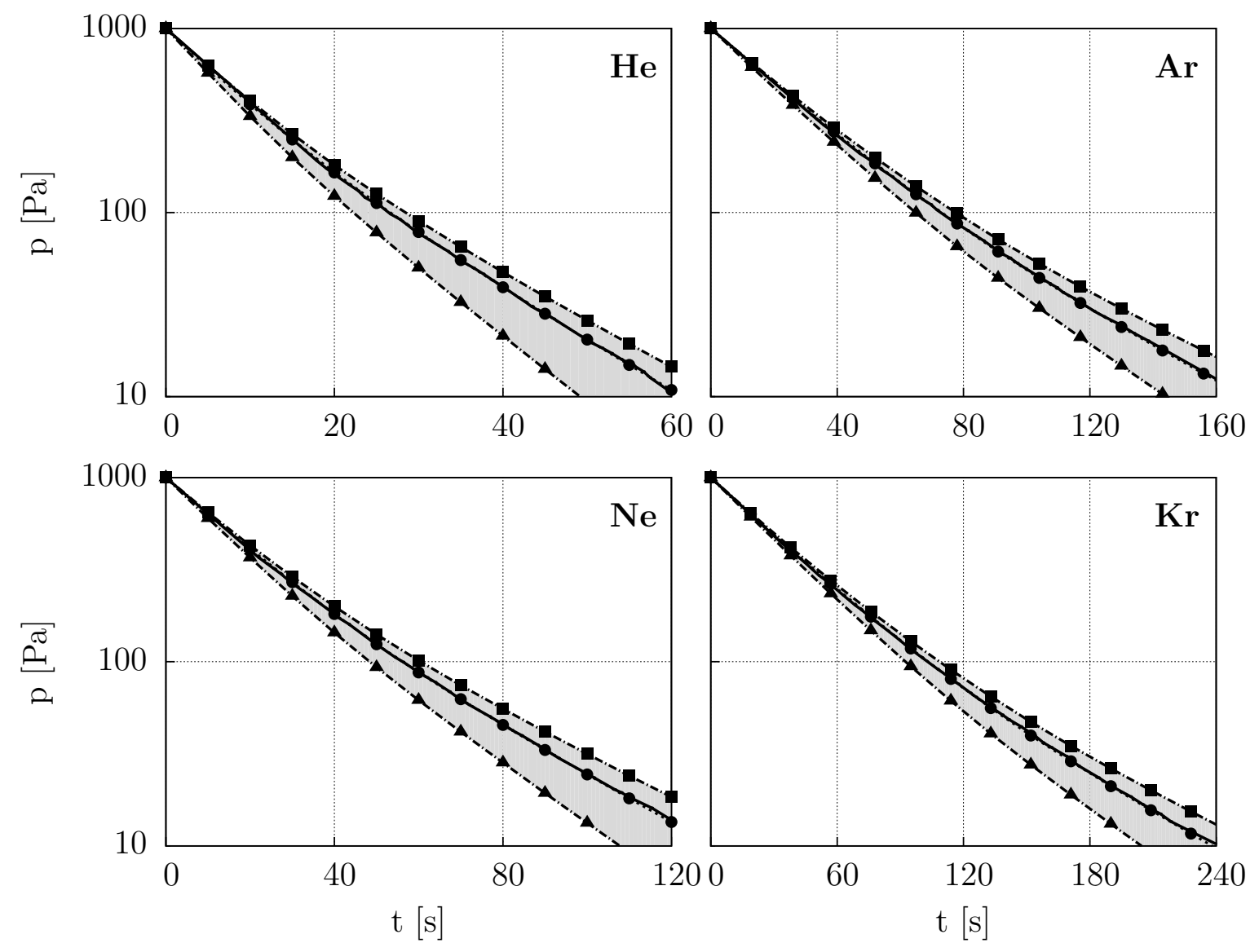

Figure 4: Temporal pressure evolution of He, Ne, Ar and $\mathrm{Kr}$ for $V=3.1 \ell$ and $R=0.50565 \mathrm{~mm}$, using different scattering kernels. Specular kernel $\left(\alpha_{t}=0\right):-\cdot \mathbf{\Delta}-\cdot \cdot($ dot-dashed line with triangles); diffuse kernel $\left(\alpha_{t}=1\right)$ : $-\cdot-\cdot$ (dot-dashed line with squares); CL kernel with $\alpha_{t}^{H e}=0.71$, $\alpha_{t}^{N e}=0.64, \alpha_{t}^{A r}=0.6, \alpha_{t}^{K r}=0.6:--\bullet--($ dashed line with circles $)$; experimental data: $-($ full line). Gray area marks the domain of possible solutions.

are only applied to a small surface area of the device. Furthermore, since specular and diffuse scattering are at the opposite ends of the accommodation spectrum, it can be argued that for the case of isothermal flow they provide the limits of the possible solution domain (grey areas in Fig. 4). 


\subsection{Uncertainty analysis of the flow field}

The results obtained for single gases are compared with respect to experimental data reported in [1] (see Fig. 4). It is easy to see that the experimental pressure profiles lie in-between the curves for specular and diffuse scattering. Thus, a better match with the experimental data can be achieved for intermediate TMAC values. For $V=3.1 \ell$ and $R=0.50565 \mathrm{~mm}$, the values which minimize the deviation from the experiment are found to be $\alpha_{t}^{\min }=0.71,0.64,0.6$ and 0.6 for $\mathrm{He}, \mathrm{Ne}, \mathrm{Ar}$ and $\mathrm{Kr}$, respectively. These values are obtained by running an optimization loop, which minimizes the discrepancy between the set of selected experimental data points and their corresponding numerical values; estimated by relying on the linear relationship of DSMC data on $\alpha_{t}$ presented in Fig. 3.

Extending the DSMC database for these values gives a significantly better agreement with the experimental results, as shown in Fig. 4. However, the obtained accommodation coefficients are unrealistically low for typical scenarios. In [18], the mean TMAC values for different monatomic gases are estimated based on the data available in the open literature and for $\mathrm{He}, \mathrm{Ne}, \mathrm{Ar}$ and $\mathrm{Kr}$ they are found to be $\alpha_{t}^{\text {mean }}=0.92,0.91,0.89$ and 0.95 , respectively, with a deviation of \pm 0.02 . Furthermore, it is argued that a value range of $0.80-1.02$ covers most of the available data for monatomic gases, irrespective of the Knudsen number and surface roughness. Further information on the TMAC values can also be found in [15] and [17].

A better match with the mean TMAC values can be obtained by taking into account the measurement uncertainties of the investigated setup. In [1], the reported values of the engineering tolerances for $L$ and $R$ are $\pm 0.01 \mathrm{~mm}$ and $\pm 0.0025 \mathrm{~mm}$, respectively. It is also specified that the expected experimental uncertainties do not exceed 2.5\%. Based on the additional information about the experimental setup

provided in [9] and [10], it is clear that a certain level of measurement uncertainty 
is associated with the upstream chamber volume as well. Therefore, if the complete procedure is repeated for $R=0.50815 \mathrm{~mm}$ and $V=3.05 \ell$ (a decrease of $1.6 \%$ ), assuming an error of $-2.5 \%$ in the initial set of experimental data, the values $\alpha_{t}^{\min }$ $=0.86,0.85,0.87$ and 0.92 are recovered for $\mathrm{He}, \mathrm{Ne}, \mathrm{Ar}$ and $\mathrm{Kr}$, respectively.
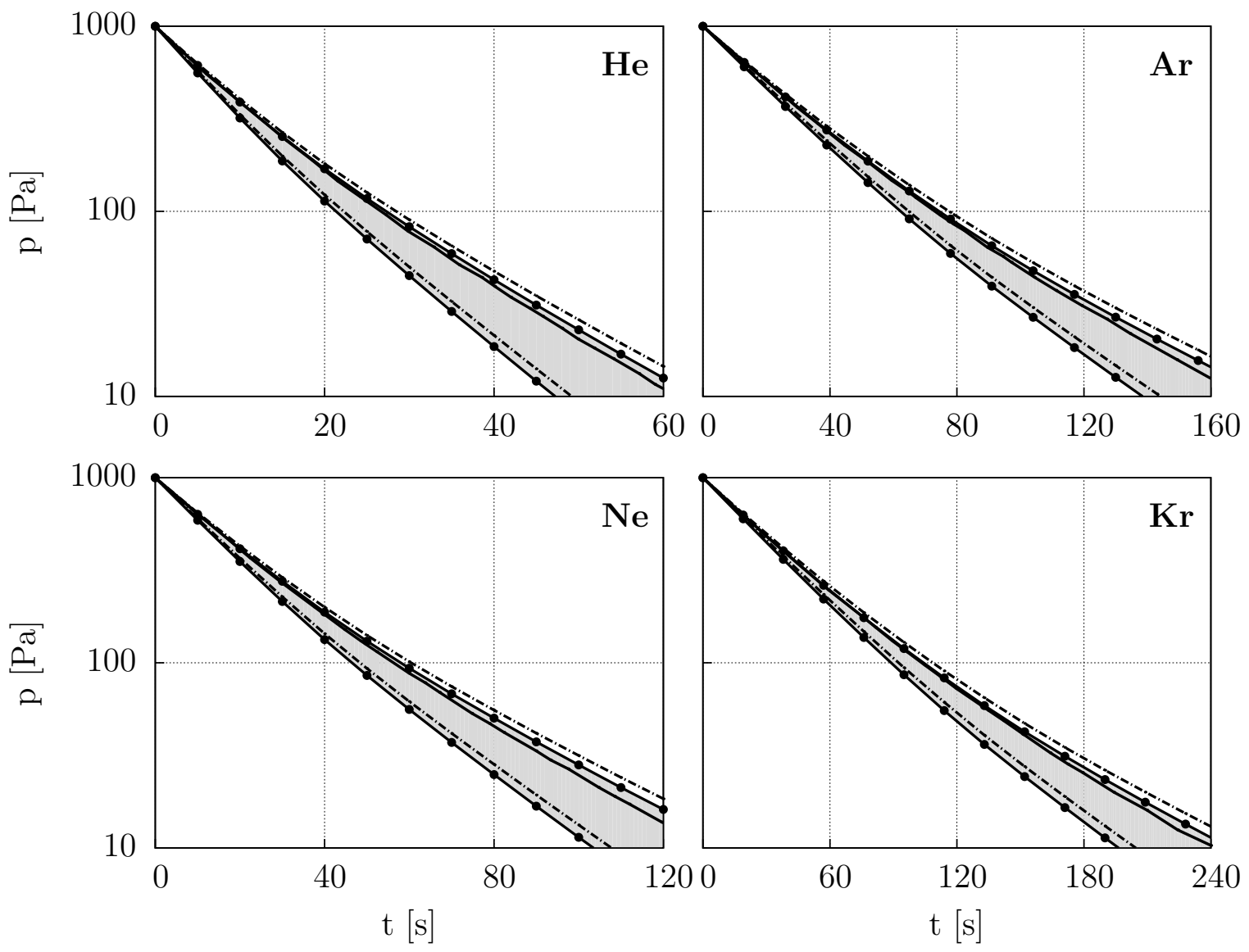

Figure 5: Shifting of the solution domain due to measurement uncertainties in the experimental setup. Boundaries of the solution domain for $V=3.1 \ell$ and $R=0.50565 \mathrm{~mm}:-\cdot-\cdot-\cdot$ (dot-dashed line); for $V=3.1 \ell$ and $R=0.51565 \mathrm{~mm}$ : - - (full line with circles). Experimental data: -(full line). Gray area marks the shifted solution domain. New values of accommodation coefficients are $\alpha_{t}^{H e}=0.88, \alpha_{t}^{N e}=0.84, \alpha_{t}^{A r}=0.88, \alpha_{t}^{K r}=0.92$. 
The performed analysis demonstrates high sensitivity of the obtained results on measurement uncertainties that can be present in the experimental setup. Due to this sensitivity, it is almost impossible to determine the correct TMAC values using the present approach, unless the exact geometrical and measurement values are known. In this specific setup however, TMAC values very close to one should be expected, especially for heavier species, since no special treatment of the tube surface has been reported. Therefore, even if the experimental results can be matched using the lower TMAC values, these are unlikely to be the cause of the observed discrepancy.

To additionally demonstrate the effect of the upstream geometry on the timedependent flow, the pressure drop is calculated for $V=3.1 \ell$ and $R=0.51565 \mathrm{~mm}$ (an increase of 2\%) as shown in Fig. 5. As a consequence of this modification, the solution domain is noticeably shifted towards shorter discharge times, but the impact of the gas-surface interaction on the resulting profiles remains unchanged. For this specific configuration, the accommodation coefficients that would minimize the discrepancy between simulation and experiment are $\alpha_{t}^{\min }=0.88,0.84,0.88$ and 0.92 for $\mathrm{He}, \mathrm{Ne}, \mathrm{Ar}$ and $\mathrm{Kr}$, respectively.

\subsection{Expansion of binary mixture and gas separation analysis}

In the next step, the same analysis was carried out for the case of binary mixture. For this purpose, the mixture of He and Ar for the specific initial values of molar fraction $C_{0}=(0.1,0.5,0.9)$ is investigated and the results for $V=3.1 \ell$ and $R=$ $0.50565 \mathrm{~mm}$ are shown in Fig. 6. The experimental pressure profiles are again located between the curves for specular and diffuse scattering. As already discussed, for this specific geometrical setup the optimal accommodation coefficients for He and Ar are

found to be $\alpha_{t}^{\min }=0.71$ and 0.6 , respectively. Implementation of these coefficients gives an improved agreement with the experimental data for all three values of $C_{0}$. 
This again highlights the importance of the gas-surface interaction in modelling of transient flows. At the same time, it confirms the validity of main assumptions (uniform pressure distribution and isothermal flow) used in the hybrid scheme.

The analysis of the gas-surface interaction also allows for the investigation of possible gas separation mechanisms. For this purpose, the temporal evolution of the molar fractions for different types of scattering kernels is given in Fig. 6, adjacent to the respective pressure profiles. It can been seen that the composition of the mixture varies significantly depending on the nature of the gas-surface interaction. For the case of specular scattering, the lighter species would discharge at the fastest rate, while the slowest rate is achieved for diffuse scattering (around 30\% lower). This information can potentially be utilized for membrane design technologies.

\section{Conclusion}

In this study, the influence of the gas-surface interaction on rarefied flow of single gases and binary mixtures into vacuum has been studied. The flow configuration consists of a large chamber connected through a short tube to vacuum, leading to a significant scale separation. Therefore, the flow is modelled using a hybrid scheme, which couples a macroscopic mass conservation equation with DSMC. The influence of gas-surface interaction has been investigated through the implementation of different scattering kernels. The results proved to be highly sensitive to changes in the tangential momentum accommodation coefficient. Based on this analysis, the accommodation coefficient values that give a good match with the experimental data have been determined. Moreover, it was shown how these values can be affected by the measurement uncertainties. In the end, the obtained results were employed to demonstrate the influence of the accommodation coefficient on the gas separation performance. 

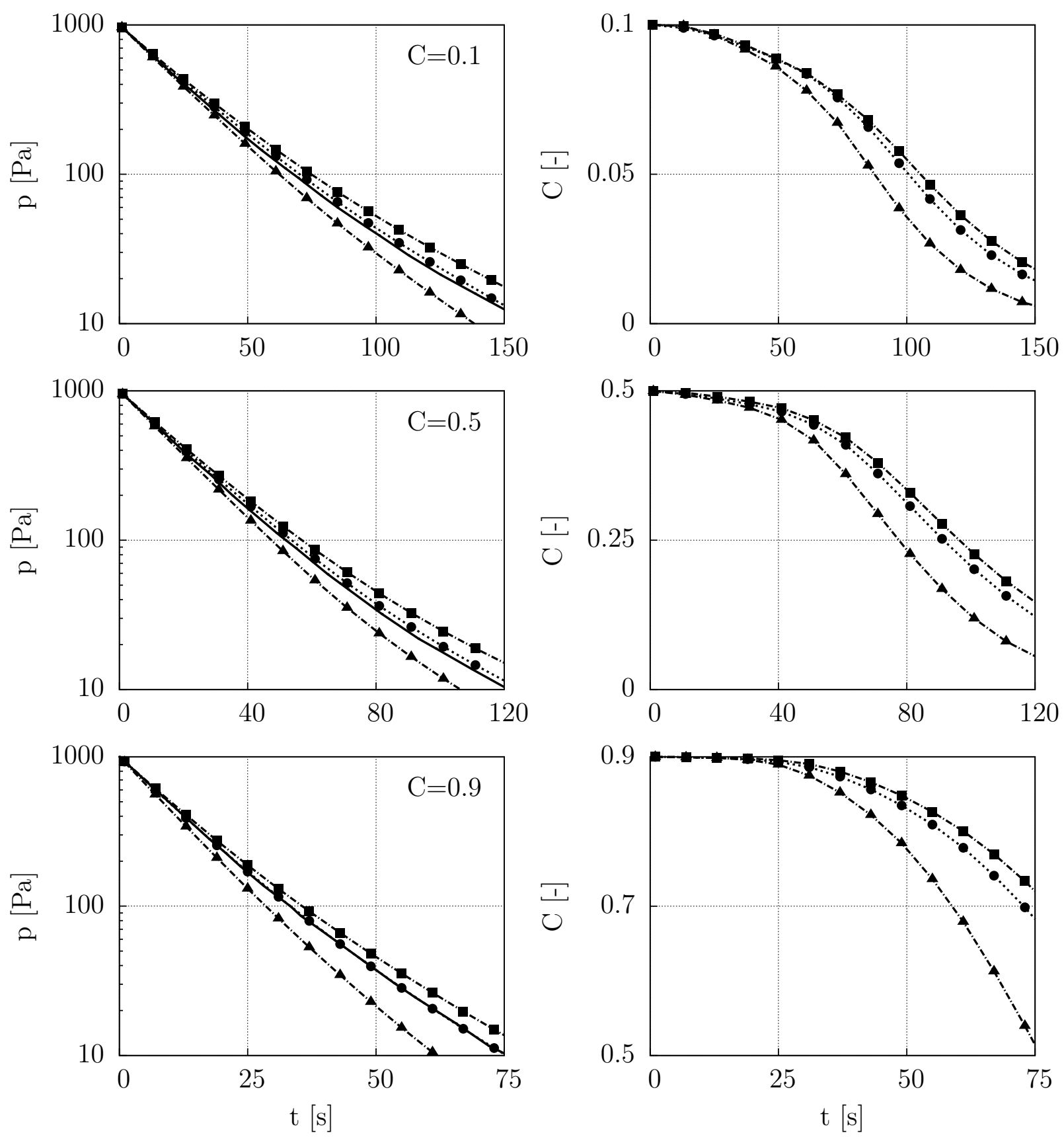

Figure 6: Temporal evolution of pressure and molar fraction in He-Ar mixture using different values of initial molar fraction, $C_{0}=(0.1,0.5,0.9)$ and different scattering kernels. Specular kernel: $-\cdot \boldsymbol{\Delta}-\cdot$ (dot-dashed line with triangles); diffuse kernel: $-\cdot \mathbf{\square}-\cdot($ dot-dashed line with squares); CL kernel with $\alpha_{t}^{H e}=0.71$ and $\alpha_{t}^{A r}=0.6$ : $--\bullet--$ (dashed line with circles); experimental data: -(full line). 


\section{Acknowledgement}

The authors would like to express sincere gratitude to Prof. Felix Sharipov for his valuable comments and suggestions on improving the quality of the paper.

Funding for this research was provided by the Swiss National Science Foundation under the grant number 153116 . 


\section{Appendix A. DSMC dataset for He-Ar mixture}

Table A.3:

DSMC database of dimensionless flow rates $J$ for He-Ar mixture as a function of rarefaction parameter $\sigma \in[0,100]$ and molar fraction $C \in[0,1]$. Gas-surface interaction is modelled with the CL kernel using $\alpha_{t}=1$ and $\alpha_{n}=1$. The upper-half contains the data for He, while the lower-half contains the data for Ar.

\begin{tabular}{|c|c|c|c|c|c|c|c|c|c|}
\hline$\sigma$ & 0 & 0.125 & 0.25 & 0.375 & 0.5 & 0.625 & 0.75 & 0.875 & 1 \\
\hline 0 & 0 & 0.0689 & 0.133 & 0.183 & 0.226 & 0.247 & 0.258 & 0.244 & 0.191 \\
\hline 0.1 & 0 & 0.0699 & 0.132 & 0.183 & 0.221 & 0.248 & 0.260 & 0.245 & 0.193 \\
\hline 0.5 & 0 & 0.0678 & 0.128 & 0.177 & 0.216 & 0.247 & 0.257 & 0.249 & 0.203 \\
\hline 1 & 0 & 0.0632 & 0.121 & 0.171 & 0.211 & 0.240 & 0.257 & 0.252 & 0.215 \\
\hline 5 & 0 & 0.0490 & 0.0969 & 0.140 & 0.181 & 0.218 & 0.249 & 0.271 & 0.271 \\
\hline 10 & 0 & 0.0448 & 0.0884 & 0.132 & 0.174 & 0.217 & 0.251 & 0.285 & 0.302 \\
\hline 25 & 0 & 0.0448 & 0.0889 & 0.132 & 0.178 & 0.222 & 0.269 & 0.308 & 0.342 \\
\hline 50 & 0 & 0.0459 & 0.0926 & 0.138 & 0.184 & 0.232 & 0.279 & 0.326 & 0.364 \\
\hline 75 & 0 & 0.0472 & 0.0945 & 0.145 & 0.191 & 0.235 & 0.288 & 0.332 & 0.378 \\
\hline 100 & 0 & 0.0484 & 0.0971 & 0.146 & 0.196 & 0.242 & 0.290 & 0.337 & 0.383 \\
\hline 0 & 0.191 & 0.156 & 0.126 & 0.0970 & 0.0705 & 0.0479 & 0.0270 & 0.0111 & 0 \\
\hline 0.1 & 0.193 & 0.161 & 0.129 & 0.100 & 0.0730 & 0.0498 & 0.0282 & 0.0116 & 0 \\
\hline 0.5 & 0.203 & 0.170 & 0.139 & 0.109 & 0.0815 & 0.0556 & 0.0333 & 0.0141 & 0 \\
\hline 1 & 0.215 & 0.182 & 0.148 & 0.119 & 0.0904 & 0.0642 & 0.0389 & 0.0176 & 0 \\
\hline 5 & 0.271 & 0.238 & 0.204 & 0.169 & 0.135 & 0.103 & 0.0680 & 0.0338 & 0 \\
\hline 10 & 0.302 & 0.266 & 0.231 & 0.195 & 0.158 & 0.119 & 0.0791 & 0.0395 & 0 \\
\hline 25 & 0.342 & 0.300 & 0.258 & 0.218 & 0.175 & 0.132 & 0.0885 & 0.0436 & 0 \\
\hline 50 & 0.364 & 0.322 & 0.277 & 0.231 & 0.185 & 0.139 & 0.0926 & 0.0457 & 0 \\
\hline 75 & 0.378 & 0.331 & 0.281 & 0.235 & 0.188 & 0.142 & 0.0951 & 0.0473 & 0 \\
\hline 100 & 0.383 & 0.335 & 0.289 & 0.242 & 0.193 & 0.145 & 0.0963 & 0.0485 & 0 \\
\hline
\end{tabular}


Table A.4:

DSMC database of dimensionless flow rates $J$ for He-Ar mixture as a function of rarefaction parameter $\sigma \in[0,100]$ and molar fraction $C \in[0,1]$. Gas-surface interaction is modelled with the CL kernel using $\alpha_{t}=0$ and $\alpha_{n}=1$. The upper-half contains the data for He, while the lower-half contains the data for Ar.

\begin{tabular}{|c|c|c|c|c|c|c|c|c|c|}
\hline$\sigma$ & 0 & 0.125 & 0.25 & 0.375 & 0.5 & 0.625 & 0.75 & 0.875 & 1 \\
\hline 0 & 0 & 0.106 & 0.193 & 0.273 & 0.328 & 0.370 & 0.383 & 0.359 & 0.283 \\
\hline 0.1 & 0 & 0.102 & 0.190 & 0.266 & 0.326 & 0.362 & 0.377 & 0.358 & 0.283 \\
\hline 0.5 & 0 & 0.0945 & 0.176 & 0.249 & 0.306 & 0.348 & 0.365 & 0.356 & 0.292 \\
\hline 1 & 0 & 0.0857 & 0.165 & 0.231 & 0.292 & 0.332 & 0.357 & 0.355 & 0.302 \\
\hline 5 & 0 & 0.0620 & 0.123 & 0.178 & 0.232 & 0.277 & 0.316 & 0.348 & 0.355 \\
\hline 10 & 0 & 0.0554 & 0.109 & 0.161 & 0.212 & 0.261 & 0.306 & 0.349 & 0.378 \\
\hline 25 & 0 & 0.0528 & 0.104 & 0.155 & 0.207 & 0.259 & 0.309 & 0.358 & 0.396 \\
\hline 50 & 0 & 0.0504 & 0.102 & 0.154 & 0.206 & 0.261 & 0.306 & 0.364 & 0.407 \\
\hline 75 & 0 & 0.0515 & 0.104 & 0.155 & 0.208 & 0.259 & 0.315 & 0.364 & 0.410 \\
\hline 100 & 0 & 0.0523 & 0.102 & 0.155 & 0.206 & 0.257 & 0.314 & 0.362 & 0.412 \\
\hline 0 & 0.283 & 0.234 & 0.186 & 0.144 & 0.105 & 0.0698 & 0.0399 & 0.0162 & 0 \\
\hline 0.1 & 0.283 & 0.234 & 0.189 & 0.145 & 0.107 & 0.0720 & 0.0416 & 0.0175 & 0 \\
\hline 0.5 & 0.292 & 0.242 & 0.198 & 0.156 & 0.117 & 0.0812 & 0.0486 & 0.0206 & 0 \\
\hline 1 & 0.302 & 0.256 & 0.212 & 0.167 & 0.129 & 0.0907 & 0.0553 & 0.0249 & 0 \\
\hline 5 & 0.355 & 0.311 & 0.266 & 0.220 & 0.176 & 0.132 & 0.0881 & 0.0432 & 0 \\
\hline 10 & 0.378 & 0.330 & 0.285 & 0.238 & 0.191 & 0.145 & 0.0968 & 0.0485 & 0 \\
\hline 25 & 0.396 & 0.348 & 0.302 & 0.252 & 0.203 & 0.153 & 0.102 & 0.0510 & 0 \\
\hline 50 & 0.407 & 0.356 & 0.304 & 0.254 & 0.203 & 0.155 & 0.102 & 0.0512 & 0 \\
\hline 75 & 0.410 & 0.362 & 0.311 & 0.261 & 0.208 & 0.156 & 0.104 & 0.0516 & 0 \\
\hline 100 & 0.412 & 0.360 & 0.310 & 0.257 & 0.205 & 0.155 & 0.105 & 0.0515 & 0 \\
\hline
\end{tabular}




\section{References}

[1] Vargas M, Naris S, Valougeorgis D, Pantazis S, Jousten K. Time-dependent rarefied gas flow of single gases and binary gas mixtures into vacuum. Vacuum 2014;109:385-396.

[2] Vargas M, Naris S, Valougeorgis D, Pantazis S, Jousten K. Hybrid modeling of time-dependent rarefied gas expansion. J Vac Sci Technol A 2014;32(2):021602.

[3] Bird GA. Molecular gas dynamics and the direct simulation of gas flows. Oxford: Oxford University Press; 1994.

[4] Sharipov F. Transient flow of rarefied gas through an orifice. J Vac Sci Technol A 2012;30(2):021602.

[5] Sharipov F. Transient flow of rarefied gas through a short tube. Vacuum 2013;90:25-30.

[6] Lihnaropoulos J, Valougeorgis D. Unsteady vacuum gas flow in cylindrical tubes. Fusion Eng and Des 2011;86(9):2139-2142.

[7] Sharipov F, Graur I. General approach to transient flows of rarefied gases through long capillaries. Vacuum 2014;100:22-25.

[8] Sonderegger K, Dür M, Buthig J, Pantazis S, Jousten K. Very fast-opening UHV gate valve. J Vac Sci Technol A 2013;31(6):060601.

[9] Jousten K, Pantazis S, Buthig J, Model R, Wüest M, Iwicki J. A standard to test the dynamics of vacuum gauges in the millisecond range. Vacuum 2014;100:1417. 
[10] Pantazis S, Jousten K. Computational and experimental study of unsteady gas flow in a dynamic vacuum standard. Vacuum 2014;109:373-384.

[11] Cercignani C, Lampis M. Kinetic models for gas-surface interactions. Transport Theor Stat 1971;1(2):101-114.

[12] Lord R. Some extensions to the Cercignani-Lampis gas-surface scattering kernel. Phys Fluids A-Fluid 1991;3(4):706-710.

[13] Gorji MH, Jenny P. A gas-surface interaction kernel for diatomic rarefied gas flows based on the Cercignani-Lampis-Lord model. Phys Fluids 2014;26(12):122004.

[14] Sharipov F. Application of the Cercignani-Lampis scattering kernel to calculations of rarefied gas flows. I. Plane flow between two parallel plates. Euro J Mech B Fluids 2002;21(1):113-123.

[15] Sharipov F. Application of the Cercignani-Lampis scattering kernel to calculations of rarefied gas flows. II. Slip and jump coefficients. Euro J Mech B Fluids 2003;22(2):133-143.

[16] Sharipov F. Application of the Cercignani-Lampis scattering kernel to calculations of rarefied gas flows. III. Poiseuille flow and thermal creep through a long tube. Euro J Mech B Fluids 2003;22(2):145-154.

[17] Sazhin O. Impact of the gas-surface scattering and gas molecule-molecule interaction on the mass flow rate of the rarefied gas through a short channel into a vacuum. J Vac Sci Technol A 2010;28(6):1393-1398.

[18] Agrawal A, Prabhu S. Survey on measurement of tangential momentum accommodation coefficient. J Vac Sci Technol A 2008;26(4):634-645. 
[19] Sharipov F. Benchmark problems in rarefied gas dynamics. Vacuum 2012;86(11):1697-1700.

[20] Sharipov F. Numerical simulation of rarefied gas flow through a thin orifice. J Fluid Mech 2004;518:35-60.

[21] Varoutis S, Valougeorgis D, Sazhin O, Sharipov F. Rarefied gas flow through short tubes into vacuum. J Vac Sci Technol A 2008;26(2):228-238.

[22] Aristov V, Shakhov E, Titarev V, Zabelok S. Comparative study for rarefied gas flow into vacuum through a short circular pipe. Vacuum 2014;103:5-8.

[23] Pantazis S, Valougeorgis D. Rarefied gas flow through a cylindrical tube due to a small pressure difference. Euro J Mech B Fluids 2013;38:114-127.

[24] Titarev V. Rarefied gas flow in a circular pipe of finite length. Vacuum 2013;94:92-103.

[25] Varoutis S, Valougeorgis D, Sharipov F. Simulation of gas flow through tubes of finite length over the whole range of rarefaction for various pressure drop ratios. J Vac Sci Technol A 2009;27(6):1377-1391.

[26] Titarev V, Shakhov E. Computational study of a rarefied gas flow through a long circular pipe into vacuum. Vacuum 2012;86(11):1709-1716.

[27] Sazhin OV, Borisov SF, Sharipov F. Accommodation coefficient of tangential momentum on atomically clean and contaminated surfaces. J Vac Sci Technol A 2013;19(5):2499-2503.

[28] Sharipov F. Gaseous mixtures in vacuum systems and microfluidics. J Vac Sci Technol A 2013;31(5):050806. 
[29] Sharipov F, Kalempa D. Gaseous mixture flow through a long tube at arbitrary Knudsen numbers. J Vac Sci Technol A 2002;20(3):814-822.

[30] Naris S, Valougeorgis D, Kalempa D, Sharipov F. Gaseous mixture flow between two parallel plates in the whole range of the gas rarefaction. Physica A 2004;336(3):294-318.

[31] Naris S, Valougeorgis D, Kalempa D, Sharipov F. Flow of gaseous mixtures through rectangular microchannels driven by pressure, temperature, and concentration gradients. Phys Fluids 2005;17(10):100607.

[32] Sharipov F, Kalempa D. Separation phenomena for gaseous mixture flowing through a long tube into vacuum. Phys Fluids 2005;17(12):127102.

[33] Maxwell JC. On stresses in rarified gases arising from inequalities of temperature. Philos T Roy Soc A 1879;p. 231-256. 